D.T. Wickramasinghe, L. Ferrario, and G.V. Bicknell, eds.

\title{
Hydrodynamical Models of Line-Driven Accretion Disk Winds
}

\author{
Timothy Kallman and Nicolas A. Pereyra
}

NASA/GSFC, Laboratory for High Energy Astrophysics, Code 662, Greenbelt, MD 20771

John M. Blondin

North Carolina State University, Department of Physics, Box 8202, Raleigh, NC 27695

The existence of winds from cataclysmic variables (CVs) has been known for some time, there are a variety of unresolved questions surrounding this phenomenon. These include: the origin of the apparent polar nature of the winds; the rate of mass loss in the winds and the associated driving mechanism; the origin for the characteristic shapes of the UV resonance line profiles, particularly the absorption component; and the apparent association between outburst state and the wind existence. Resolution of all these issues depends on understanding the dynamics of the wind. In this paper we describe one and two dimensional models for disk wind dynamics.

Using $1 \mathrm{D}$ analytic models we have explore the physical conditions necessary for the existence of a disk wind, and study the dependence of wind speed and mass loss rate on radius. This leads to a criterion which must be satisfied by the rate of increase in the radiation field with height above the disk surface and by the parameters describing the line radiation pressure force (c.f. Castor, Abbott and Klein, 1976).

The 2D models are calculated using the piecewise parabolic method for the solution of the hydrodynamic equations. A sample calculation of the wind density and velocity distribution is shown in the figure. Resonance line profiles have also been calculated for this simulation. The results are consistent with the observed properties of CVs in apparent the polar nature of the wind, the location of the maximum absorption at roughly half the terminal speed of the P-Cygni profiles and the order of magnitude of the terminal speeds. The $2 \mathrm{D}$ models show that centrifugal forces produce a region of compressed gas and shocks in the wind. If this region were absent, the line optical depths would be too low to produce the optical depths required to explain the P-Cygni profile of CVs. Our models do not show strong time variability. These results are described in more detail in two journal articles which are in preparation (Pereyra, Kallman, and Blondin, 1997).

\section{References}

Castor, J., Abbott, D., and Klein, R. 1975, ApJ, 195, 157

Pereyra, N. A., Kallman, T., and Blondin, J.,1997, ApJ, in press 


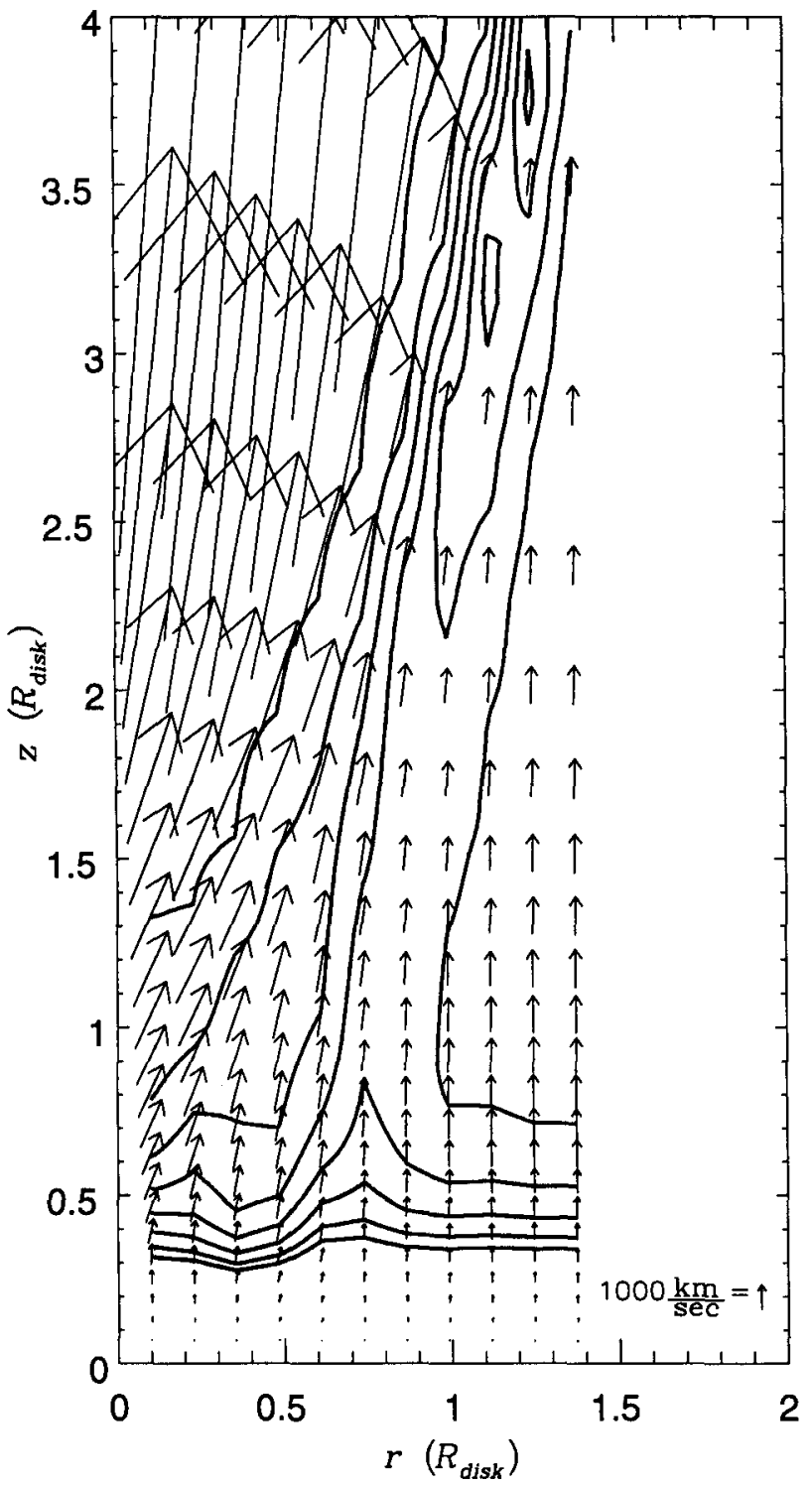

Figure 1. Vector field graph of wind velocity superimposed with a contour graph of wind density for a two dimensional model 